\title{
Human FPR2/ALX receptors are highly expressed in septic patients and regulate autophagy in PMA-stimulated neutrophils
}

\author{
Yao Lu, Han Zhang, Wenjun Xia, Guixiang Sun, Lingfeng Wang, Linjing Zhang, Aiqing Wen* \\ Department of Blood Transfusion, Research Institute of Surgery, Daping Hospital, Army Medical University, Chongqing 400042, China
}

\begin{abstract}
Formyl peptide receptor 2-lipoxin receptor (FPR2/ALX) and its agonists are well-defined mechanisms in antiinflammatory and pro-resolving response, and neutrophils actively participate in inflammation. However, FPR2/ ALX expression in neutrophils and the effects of FPR2/ALX agonist in autophagy of neutrophils under inflammatory circumstances are not fully understood. In this study, flow cytometric analysis and real-time PCR were used to detect the protein and mRNA expression of FPR2/ALX in neutrophils in healthy volunteers and septic patients. The effects of FPR2/ALX agonist BML-111 alone or with pro-inflammatory stimulant in neutrophils were assessed by Western blot. The results showed that both protein and mRNA expression of FPR2/ALX in neutrophils in patients with sepsis were significantly increased compared with that in healthy subjects $(P<0.05)$. PMA promoted the conversion of LC3- I to LC3- II in neutrophils, a key marker of autophagy. BML-111 alone had no effect on autophagy in neutrophils. Nevertheless, BML-111 reduced PMA-induced LC3 processing in neutrophils. Our results indicated that FPR2/ALX expression increased in neutrophils in septic patients. FPR2/ALX agonist BML-111 reduced LC3 processing in neutrophils with pro-inflammatory stimulation. These findings demonstrated a novel effect of FPR2/ALX activation in regulating autophagy.
\end{abstract}

Keywords: sepsis, FPR2/ALX, BML-111, LC3

\section{INTRODUCTION}

Sepsis, a life-threatening clinical organ dysfunction syndrome caused by dysregulated host response to infection ${ }^{[1]}$, is a major cause of mortality and critical illness worldwide ${ }^{[2]}$, and its incidence is increasing ${ }^{[3]}$. Sepsis is now recognized to involve early activation of both pro- and anti-inflammatory responses, and endogenous anti-inflammatory mediators are present to ensure the stabilization of the host response, among which lipoxin A4 (LXA4) and annexin Al are the best studied. Pro-resolving mediators like LXA4 play an

*Correspondence to: Aiqing Wen, MD, PhD, Department of Blood Transfusion, Research Institute of Surgery, Daping Hospital, Army Medical University. NO 10, Changjiang Branch Road, Daping District, Chongqing 400042 , China. TEL: +86-23-68757651, E-mail: dpyysxkwaq@hotmail.com. Conflicts of interest: The authors declare that they have no conflict of interest. important role in the resolution of inflammation via binding to formyl peptide receptor 2-lipoxin receptor (FPR2/ALX), which has been shown to exert regulatory and organ protective functions in sepsis ${ }^{[4,5]}$.

Neutrophils have a pivotal role in sepsis as one of the first line of innate immune defense. These cells migrate from the circulating blood to the infected area to engulf and eliminate microorganisms via phagocytosis, degranulation, production of reactive oxygen species (ROS), antimicrobial peptides secretion and the formation of neutrophil extracellular traps $(\mathrm{NETs})^{[6]}$. After performing their anti-microbial action, these cells undergo apoptosis and are phagocytized by macrophages, allowing the recovery of the balance of immune defense. Since the excessive and improper activation of neutrophils could cause tissue damage and participate in the pathogenesis of organ dysfunc- 
tion in sepsis ${ }^{[7]}$. Therefore resolving inflammation is of utmost importance to avoid host damage.

FPR2/ALX has been shown to be one of the receptors involved in the resolution of inflammation. Growing evidence indicates that the activation of FPR2/ALX by pro-resolving mediators regulates the function of neutrophils. FPR2/ALX agonist LXA4 regulates neutrophil-platelet aggregation in inflammatory diseases ${ }^{[8,9]}$, affects the formation of NETs ${ }^{[10]}$, increases bacteria clearing function ${ }^{[11]}$, attenuates tumor necrosis factor (TNF-a)-initiated neutrophil responses and trafficking ${ }^{[12]}$, and down-regulates proinflammatory gene expression. These studies indicated that this anti-inflammatory receptor and its pro-resolving agonists serve regulatory functions in neutrophils during the host defense response. Even though, the effect of pro-resolving mediators on neutrophils remains to be completely elucidated.

Autophagy is the major intracellular degradation system, which is characterized by the formation of double-membrane autophagosomes. A key marker of autophagosome formation is the proteolytic cleavage of cytoplasmic microtubule associated protein 1 light chain 3 (LC3) to generate LC3- I which then conjugates to phosphatidylethanolamine to form LC3- II . LC3- II is then recruited and incorporated into the autophagosomal membrane ${ }^{[13]}$. Autophagy plays a multifunctional role in infection, inflammation and immunity, by regulating interactions with innate immune signaling pathways, removing endogenous inflammasome agonists and affecting the secretion of immune mediators ${ }^{[14]}$. Recent studies have shown that autophagy regulates neutrophil degranulation, and plays an important role in the inflammatory activity of neutrophils ${ }^{[15]}$ and pro-resolving lipid mediators 15-epi-LXA4. The resolvin D1 improves both survival and functionality of macrophages by activating autophagy ${ }^{[16]}$. Nevertheless, whether LXA4 regulates autophagy in neutrophils under inflammation conditions has not been addressed.

In the present study, we aim to determine the expression of FPR2/ALX in neutrophils in patients with sepsis and find whether FPR2/ALX activation by proresolving mediators regulates autophagy in neutrophils with inflammatory stimulation. FPR2/ALX expression in neutrophils from sepsis patients up-regulated compared with that from healthy subjects. The neutrophils isolated from healthy volunteers with the stimulation of phorbolmyristate acetate (PMA) were used as a cell model to study whether pro-resolving mediators affect autophagy in neutrophils in vitro. Here for the first time, the study showed that the activation of FPR2/ALX by adding a LXA4 analogue 5(S) and
6(R)-7-trihydroxymethyl heptanoate (BML-111) decreased LC3 processing in neutrophils. These findings provided new evidence that pro-resolving mediators exerted a regulating role in neutrophils via FPR2/ALX under inflammation conditions.

\section{MATERIALS AND METHODS}

\section{Sample collection and preparation}

The study protocol was in accordance with the Declaration of Helsinki and has been approved by the Ethics Committee of Daping Hospital, Army Medical University. All blood samples were obtained after informed consent. Samples of patients with sepsis were collected within 24 hours after diagnosis of sepsis by doctors in Daping Hospital's intensive care unit (ICU). The diagnosis of sepsis and severe sepsis in our study was based on the definition of Sepsis-3 (Third International Consensus Definition for Sepsis and Septic Shock $)^{[1]}$. Patients with infection and infection-induced organ dysfunction [a Sequential Organ Failure Assessment (SOFA) score of 2 points or more] were enrolled in the study. Healthy control samples were collected from healthy volunteers. Peripheral venous blood was collected aseptically from each subject into ethylenediamine tetraacetic acid (EDTA) anticoagulant tubes. Each sample was numbered and de-identified.

\section{Isolation of neutrophils}

Neutrophils were isolated from peripheral blood samples by centrifugation with separation medium (Haoyang, Tianjin, China) within $2 \mathrm{~h}$ after collection, according to the manufacturer's instructions. Briefly, the separation medium and peripheral blood were carefully added into a $15 \mathrm{~mL}$ centrifuge tube respectively to form a two layer column and then centrifuged at $600 \mathrm{~g}$ for $30 \mathrm{~min}$ at $22{ }^{\circ} \mathrm{C}$. The white supernatant layer (which is rich in neutrophils) was collected and treated with red blood cell lysing buffer (Beyotime, Shanghai, China) to remove the residual red blood cells. After 3 washes with washing buffer, neutrophils were resuspended in RPMI 1640 medium (Hyclone, Utah, USA) which was supplemented with $10 \%$ fetal bovine serum, and then counted using a cell count plate. Neutrophil purity ( $>98 \%$ ) was checked by CD15 fluorescence combined with side scatter and viability $(>95 \%)$ by trypan blue staining.

\section{Flow cytometric analysis for FPR2/ALX expres- sion in neutrophils}

Totally $50 \mu \mathrm{L}$ fresh whole blood was treated with red blood cell lysing buffer and centrifuged to harvest 
cells. Fc-block cells with blocking IgG for 15 min at room temperature were incubated with FITC conjugated anti-hFPRL1/FPR2 antibody (R\&D systems, USA) for 30 minutes in the dark. After two washes with flow cytometry staining buffer, cells were resuspended in the buffer and analyzed on a BDAccuri C6 flow cytometery. Data were analyzed using FlowJo 7.6 software (BD Biosciences). Mouse IgG FITC-conjugated control antibody was used as isotype control to confirm the specificity of the procedure. Neutrophils were gated by using forward and side scatter and analyses were carried out on 20,000 events for each sample. Results were expressed as the percentage of positive cells. Median fluorescence intensity (MFI) was also recorded for analysis.

\section{Real-time reverse transcriptase-polymerase chain reaction (RT-PCR) for FPR2/ALX ex- pression}

The total RNA was extracted from neutrophils and quantified. Reverse-transcription was performed with PrimeScript $^{\mathrm{TM}}$ RT reagent Kit with gDNA Eraser (Takara Bio Inc, Japan) and cDNA was amplified by real-time PCR with SYBR ${ }^{\circledR}$ Green Realtime PCR Master Mix (Toyobo Co, Osaka, Japan). The sequences of primers were as follows: human $\beta$-actin, forward 5 '-CTGGGACGACATGGAGAAAA-3' and reverse 5'-GGCTGGGGTGTTGAAGGTCT-3'; human FPR2, right 5'-GCCAGCAGACTCATAGGACA-3' and left 5'-GCACACAGGAAAAGGAGCTT-3'. Relative expression levels were determined with the comparative cycle threshold $\left(\mathrm{C}_{\mathrm{T}}\right)$ method for relative gene expression quantification against $\beta$-actin.

\section{Western blot for LC3 expression}

Human neutrophils $\left(3 \times 10^{6}\right.$ cells $\left./ \mathrm{mL}\right)$ were suspended in RPMI and incubated with $25 \mathrm{nmol} / \mathrm{L}$ PMA for $30 \mathrm{~min}$ at $37^{\circ} \mathrm{C}$ under a $5 \% \mathrm{CO}_{2}$ atmosphere. Then, the cells were harvested and whole-cell lysates were prepared. The protein concentrations were determined using a bicinchoninic acid (BCA) protein assay kit (Beyotime, Shanghai, China). The lysates (20 to $40 \mu \mathrm{g}$ of each sample) were separated by sodium dodecyl sulfate $8 \%$ 12\% polyacrylamide gel electrophoresis, and transferred to a polyvinylidene fluoride membrane for $90 \mathrm{~min}$ at $110 \mathrm{~V}$ in a blotting system (Bio-Rad, USA). Membranes were placed in blocking buffer ( $5 \%$ no fat dry milk), and incubated overnight at $4{ }^{\circ} \mathrm{C}$ with corresponding antibodies. To ensure equivalent amounts of protein in each lane, rabbit polyclonal antibody to GAPDH $(1: 1,000)$ was used as internal loading control. Detection was achieved us- ing swine anti-rabbit HRP-linked secondary antibody (Cell Signaling Technology, USA), followed by Immobilon ${ }^{\mathrm{TM}}$ Western chemilumenescent HRP substrate (Millipore, USA). Immunoreactive bands were visualized and quantified by Bilber Fusion Fx Instruments (BilberLourmat, France).

\section{Statistical analysis}

Statistical analyses were performed using SPSS Statistics 19.0 (IBM SPSS, Chicago, IL, USA). Continuous variables were summarized as means and SDs, or medians and interquartile ranges (IQRs), as appropriate to each measure. Categorical variables were summarized as counts and percentages. Statistical analysis was performed using the independent Student $t$ test or $\chi^{2}$ test as appropriate. $P<0.05$ was defined as statistically significant.

\section{RESULTS}

\section{Patient characteristics and neutrophil parameters}

A total of 27 septic patients and 15 healthy subjects were enrolled in the study. The clinical characteristics and neutrophil parameters were summarized in Table 1. The septic patients' mean SOFA score was 7.6, and nearly half of them were identified as severe sepsis, suggesting severe organ dysfunction in these patients. The 27 septic patients included 11 patients with underlying abdominal infection, 5 patients with trauma, 5 patients with respiratory tract infection, 3 patients with infection of the urinary tract, 3 patients with primary bacteremia, and 1 patient with maxillofacial infection.

Table 1 Summary of patient characteristics and neutrophil parameters

\begin{tabular}{lcc}
\hline Characteristics & Healthy control $(n=15)$ & Septic patients $(n=27)$ \\
\hline Demographic profile & & \\
Age[years, median(IQR)] & $50(39 \sim 60)$ & $62(57 \sim 73)$ \\
Male[n(\%)] & $10(66.7)$ & $18(66.7)$ \\
SOFA score $(\operatorname{mean} \pm$ SD) & - & $7.6 \pm 3.9$ \\
Severe sepsis $[n(\%)]$ & - & $13(48.1)$ \\
Infectious diagnosis $[n(\%)]$ & & \\
Abdominal infection & - & $11(40.7)$ \\
Trauma & - & $5(18.5)$ \\
Pneumonia & - & $5(18.5)$ \\
Urinary tract infection & - & $3(11.1)$ \\
Blood stream infection & - & $3(11.1)$ \\
Maxillofacial infection & - & $1(3.7)$ \\
Neutrophil parameters(mean \pm SD) & & \\
PMN count $\left(\times 10^{9} / \mathrm{L}\right)$ & $5.7 \pm 1.4$ & $14.4 \pm 8.8^{*}$ \\
PMN/WBC ratio(\%) & $60.4 \pm 6.8$ & $82.6 \pm 12.1^{*}$ \\
\hline
\end{tabular}

IQR: interquartile range; $n$ : number; SOFA: sequential organ failure assessment; SD: standard deviation; PMN: polymorphonuclear leukocyte. ${ }^{*} P<0.05$, septic patients $v s$. healthy control. 
Among these patients, 1 patient had both respiratory infection combined with abdominal infection. Both polymorphonuclear leukocyte (PMN) count and PMN/ white blood cell ratio were significantly higher than that in the healthy subjects $(P<0.05)$.

\section{Up-regulation of neutrophil FPR2/ALX ex- pression in patients with sepsis}

Previous publications have shown that FPR2/ALX expression in neutrophils may change in certain inflammation diseases, like preeclampsia ${ }^{[17]}$ and severe asthma $^{[18]}$. To evaluate whether this receptor expression is up- or down-regulated in sepsis, the FPR2/ ALX expression in the neutrophils of sepsis samples and healthy samples was detected(Fig.1). The neutrophils' purity was detected by flow cytometric analysis immediately after isolation from whole blood and was found to be $>98 \%$ in each sample. The mRNA expression of FPR2/ALX in neutrophils separated from peripheral blood was detected by real-time PCR and was found significantly up-regulated in patients with sepsis compared with healthy subjects, as shown in
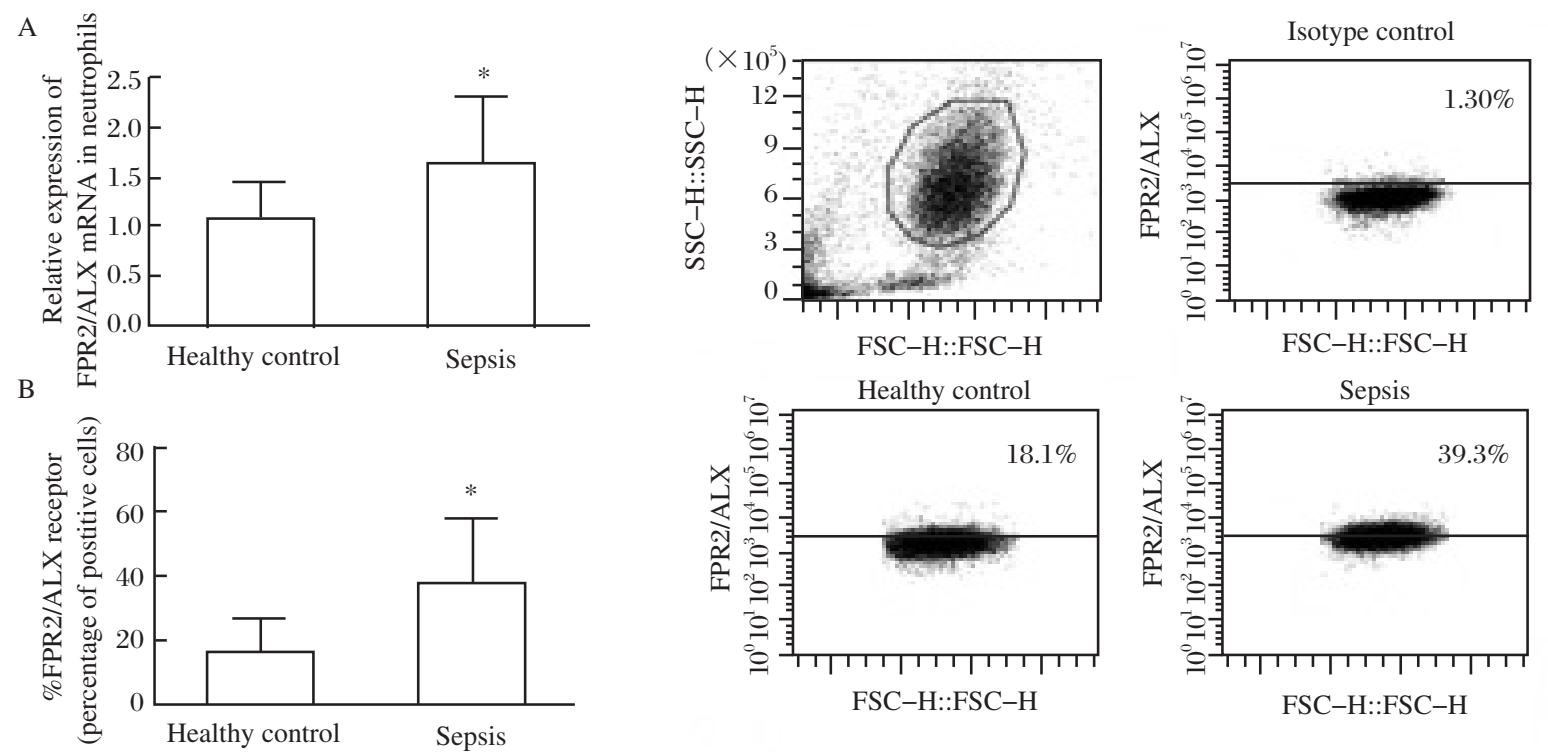

Fig. 1 The expression of FPR2/ALX increased in neutrophils of septic patients. (A)The mRNA expression of FPR2/ ALX in neutrophils isolated from patients with sepsis and healthy control were detected by real-time $\mathrm{PCR}\left(n=15\right.$; ${ }^{*} P<0.05 v s$. control); (B) Percentage of FPR2/ALX positive neutrophils in patients with sepsis and healthy controls by flow cytometric analysis $(n=13$; ${ }^{*} P<0.05$ vs. control).

Fig. 1A. Similar changes in neutrophil FPR2/ALX at protein levels were also confirmed by using flow cytometric analysis (Fig. 1B).

\section{BML-111 decreased LC3 processing in PMA- stimulated neutrophils}

To evaluate whether pro-resolving mediator plays a role in neutrophil autophagy under inflammation conditions, neutrophils were pre-incubated with different concentrations of BML-111 before PMA stimulation. As pro-resolving lipid mediators like 15-epi-LXA4 and resolvin Dl can activate autophagy in macro phages ${ }^{[16]}$, whether pro-resolving agonist BML-111 activates autophagy in neutrophils was firstly tested. The treatment with BML-111 in neutrophils, or a relatively high concentration of a stable LXA4 analogue, did not affect autophagy level, which was revealed by LC3- II expression, an autophagy marker. As shown in Fig.2, $25 \mathrm{nmol} / \mathrm{L}$ PMA induced a significantly in- crease in the formation of LC3- II . Pretreatment with BML-111 for 30 minutes before stimulation with PMA caused a decrease of LC3- II expression. These findings indicated that BML-111 alone did not decrease autophagy in neutrophil, but could decrease PMA-induced autophagy in neutrophils via the activation of FPR2/ALX.

\section{DISCUSSION}

The present work contributes with several important findings. First, the FPR2/ALX expression in neutrophils in sepsis patients increased significantly compared to that in healthy controls. Second, the activation of FPR2/ALX decreased autophagy induced by PMA. Moreover, the study showed a functional interplay of FPR2/ALX and autophagy mechanisms ex vivo, i.e., FPR2/ALX agonist BML-111 caused a significant reduction of LC3 processing. Our results indicated the possible mechanism of pro-resolving 
PMA-stimulated neutrophils, 2018, 2(3)

A

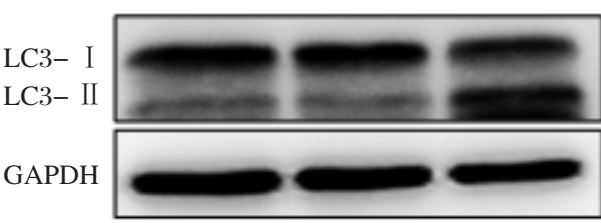

B

Healthy PMA

$\operatorname{PMA}+\mathrm{BML}(\mu \mathrm{md} / \mathrm{L})$

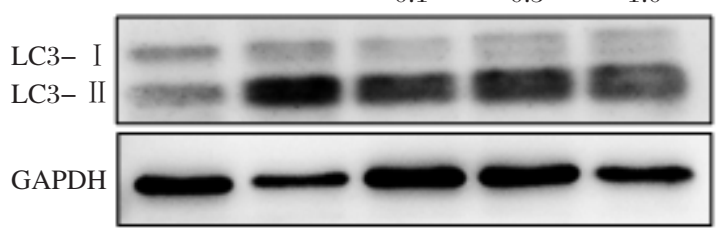

Fig.2 FPR2/ALX agonist BML-111 inhibited PMA-induced LC3 processing. (a)Neutrophils isolated from healthy volunteers were treated with vehicle or $25 \mathrm{nmol} / \mathrm{L}$ PMA or BML-111 $1 \mu \mathrm{mol} / \mathrm{L}$ for $30 \mathrm{~min}$, and then the protein levels of LC3- I and LC3 - II were detected by using Western blot. (b)Neutrophils isolated from healthy volunteers were treated with vehicle or 25 nmol/L PMA for $30 \mathrm{~min}$ in the presence or absence of a pretreatment with different concentrations of BML-111, FPR2/ALX agonist, for $30 \mathrm{~min}$, and then the protein levels of LC3- I and LC3- II were detected by using Western blot.

mediators like BML-111 on innate host defense in sepsis via regulating neutrophil function.

The FPR2/ALX receptor belongs to a family of $\mathrm{G}$-protein coupled receptors which is involved in the process of resolution of inflammation ${ }^{[19]}$. FPR2/ ALX receptors are expressed in neutrophils, monocytes, microglias and some other tissues like lung and liver ${ }^{[20]}$. The increased FPR2/ALX expression in neutrophils was found in sepsis patients. FPR2/ ALX expression is induced by both pro-inflammatory stimuli and pattern recognition receptors as well as anti-inflammatory signaling. It has been reported that TNF- $a$ and bacterial endotoxin enhanced cell surface FPR2/ALX receptor expression ${ }^{[4]}$.

LXA4 was the first endogenous lipid ligand to be identified which exerts its anti-inflammatory and proresolving effects by binding FPR2/ALX with high affinity. It has been reported that LXA4 can reduce neutrophil migration but increase neutrophil bacteria clearing function ${ }^{[11]}$. However, the mechanism of how LXA4 exerts its function on neutrophil has not been studied sufficiently. Autophagy plays a multifunctional role in infection, inflammation and immunity ${ }^{[14]}$, and recent studies have shown that autophagy plays an important role in the inflammatory activity of neutrophils ${ }^{[15]}$. Here, the study demonstrated that BML-111, a LXA4 analogue, reduced LC3 processing, which was induced by PMA. It has been reported that 15-epi-LXA4 and resolvin Dl (both of which are agonists of FPR2/ALX) can promote autophagy in murine and human macrophages. When stimulated with PMA, pretreating with BML-111 significantly decreased LC3 processing (Fig.2B). However, in our study, BML-111 alone had no effect on autophagy in human neutrophils (Fig. 2A).

In summary, our study demonstrated that FPR2/ ALX expression in neutrophils separated from septic patients was significantly higher than that from healthy volunteers. Also, our study demonstrated that FPR2/ALX activation by its agonist BML-111 could inhibit LC3 processing in neutrophils with inflammatory stimulation, indicating a new role for FPR2/ ALX agonist in regulating intracellular autophagy. The present study provides a new insight for regulating innate immune response with pro-resolution mediators.

\section{Abbreviations}

LXA4, lipoxin A4; FPR2/ALX, formyl peptide receptor 2-lipoxin receptor; ROS, reactive oxygen species; NETs, neutrophil extracellular traps; TNF-a, tumor necrosis factor $\mathrm{a}$; LC3, microtubule-associated protein 1 light chain 3; PMA, phorbolmyristate acetate; BML-111, 5(S), 6(R), 7-trihydroxyheptanoic acid methyl ester; ICU, intensive care unit; SOFA, sequential organ failure assessment; EDTA, ethylenediamine tetraacetic acid; MFI, median fluorescence intensity; RT-PCR, real-time reverse transcriptasepolymerase chain reaction; BCA, bicinchoninic acid; IQR, interquartile range; $\mathrm{SD}$, standard deviation; PMN, polymorphonuclear leukocyte.

\section{Acknowledgments}

This work received the grant support of the National Natural Science Foundation of China (81171790).

\section{References}

[1] Singer M, Deutschman CS, Seymour CW, et al. The third internationalconsensus definitions for sepsis and septic shock(Sepsis-3). JAMA, 2011, 315(8):801-10.

[2] Vincent JL, Marshall JC, Namendys-Silva SA, et al. Assessment of the worldwide burden of critical illness: the Intensive Care Over Nations (ICON) audit. Lancet Respir Med, 2014, 2(5):380-6.

[3] Gaieski DF, Edwards JM, Kallan MJ, et al. Benchmarking the incidence andmortality of severe sepsis in the United States. Crit Care Med,2013,41(5):1167-74.

[4] Gobbetti T, Coldewey S, Chen J, et al. Nonredundant protective properties of FPR2/ALX inpolymicrobial murine sepsis. Proc Natl Acad Sci U S A, 2014,111(52):18685-90.

[5] Cheng Q, Wang Z, Ma R, et al. Lipoxin A4 protects 
against lipopolysaccharide-induced sepsis by promoting innate response activator B cells generation. Int Immunopharmacol,2016, 39:229-35.

[6] Brinkmann V, Reichard U, Goosmann C, et al. Neutrophil extracellular traps kill bacteria. Science, 2004,303(5663):1532-5.

[7] Czaikoski PG, Nascimento DC, Sônego F, et al. Neutrophil extracellular traps induce organ damage during experimental and clinical sepsis. PLoS One,2016,11(2): e0148142.

[8] Ortiz-Muñoz G, Mallavia B, Bins A, et al. Aspirin-triggered 15-epi-lipoxin A4 regulates neutrophil-platelet aggregation and attenuates acute lunginjury in mice. Blood, 2014,124(17):2625-34.

[9] Vital SA, Becker F, Holloway PM, et al.Formyl-peptide receptor 2/3 lipoxin A4 receptor regulates neutrophilplatelet aggregation andattenuates cerabral inflammation. Circulation, 2016, 133(22):2169-79.

[10] Tibrewal S, Ivanir Y, Sarkar J, et al. Hyperosmolar stress induces neutrophil extracellular trap formation:Implications for dry eye disease. Investigative Ophthalmology \& Visual Science, 2014,55(12): 7961-69.

[11] Wu B, Walker J, Spur B, et al. Effects of lipoxin A4 on antimicrobial actions of neutrophils in sepsis. Prostaglandins Leukot Essenti Fatty Acids,2015,94:55-64.

[12] Pouliot M, Serhan CN. Lipoxin A4 and aspirin-triggered 15-epi-LXA4 inhibit tumornecrosis factor- $\alpha$-initiated neutrophil responses and trafficking: novel regulators of acytokine-chemokine axis relevant to periodontal diseases. J Periodontal Res,1999,34(7):370-3.

[13] Tanida I, Ueno T, Kominami E. LC3 and autophagy. Methods Mol Biol, 2008,445:77-88.

[14] Deretic V, Saitoh T, Akira S. Autophagy in infection, inflammation and immunity. Nat Rev Immunol, 2013,13(10):722-37.

[15] Bhattacharya A, Wei Q, Shin JN, et al. Autophagy is required for neutrophil-mediated inflammation. Cell Rep, 2015,12(11):1731-39.

[16] Prieto P, Rosales-Mendoza CE, Terrón V, et al. Activation of autophagy inmacrophages by pro-resolving lipid mediators. Autophagy, 2015,11(10):1729-44.

[17] Xu Z, Zhao F, Lin F, et al. Preeclampsia is associated with a deficiency of lipoxin A4, an endogenous anti-inflammatory mediator. Ferti Steril, 2014, 102(1):282-90.

[18] Gagliardo R, Gras D, La Grutta S, et al. Airway lipoxinA4/ formyl peptide receptor 2-lipoxin receptor levels in pediatric patients with severe asthma. J Allergy Clin Immunol, 2016,137(6):1796-806.

[19] Corminboeuf O, Leroy X. FPR2/ALXR agonists and the resolution of inflammation. J Med Chem, 2014, 58(2):537-59.

[20] Dufton N, Perretti M.Therapeutic anti-inflammatory potential of formyl-peptide receptoragonists. Pharmacol Ther, 2010, 127(2):175-188.

(Received 01 August 2018, Revised 03 September 2018, Accepted 09 September 2018) 